\title{
Perfil psicosocial del incendiario forestal español privado de libertad.
}

\author{
Spanish Forest Arsonists Inmates Psychosocial Profile
}

\author{
José Luis González Álvarez ${ }^{1}$ \\ Verónica Muñoz Espinosa ${ }^{2}$ \\ $\mathrm{M}^{a}$ Luisa Calcerrada Alcázar ${ }^{3}$ \\ Andrés Sotoca Plaza ${ }^{3}$
}

(1) Secretaría de Estado de Seguridad. Gabinete de Coordinación y Estudios, Madrid, España.

(2) Universidad Complutense de Madrid, Madrid, España.

(3) Guardia Civil. Unidad Técnica de Policía Judicial. SACD, Madrid, España.

Email correspondencia:me.criminologa@gmail.com

\section{Resumen}

Sólo existen dos líneas de investigación acerca del incendiario forestal, una portuguesa y otra española, que mediante la técnica del perfilamiento criminal han obtenido una tipología que informa sobre qué tipo de persona cometerá con mayor probabilidad un incendio forestal. A pesar de los avances, la tasa de esclarecimiento en este delito es todavia baja, por lo que este estudio trata de ayudar a una mejora de la investigación policial mediante el estudio de las características psicosociales de 50 incendiarios forestales privados de libertad, constituyendo un estudio pionero pues nadie se ha centrado hasta ahora en los que llegan a prisión. Los resultados acerca de su perfil muestran que predomina una baja inteligencia, consumen drogas o alcohol, han estado en tratamiento psicológico previo y destaca como motivo del incendio padecer algún tipo de trastorno. Dichos resultados dejan entrever que estas carencias podrian ser las que hacen que sean efectivamente descubiertos, ingresando en prisión.

Palabras Clave: Incendiario forestal, incendiario, perfil psicológico, perfilamiento criminal, población carcelaria

\begin{abstract}
Only two research areas exist about the forest arsonist, one is portuguese and the other one is Spanish, which, by the criminal profiling technique, have obtained a typology which gives information about if some kind of people would most likely commit a forest arson. In spite of advances, the clarification rate in this type of crime is still low because of that this research try to help in order to improve the police investigation by studying the psychosocial characteristics of 50 forest arsonists inmates, beeing a pioneering research since nobody has studied until this moment those who finally become inmates. The results about the profile show that is typical a low intelligent, they normally take drugs or alcohol, received pshicological treatment, and regarding to the arson motive, "suffering from any disorder" stands out. These results let us to catch a glimpse of the fact that these deficiencies might make them be discovered, beeing deprived of freedom.
\end{abstract}

Keywords: Forest arsonist, arsonist, psychosocial profile, criminal profiling, inmates

Fecha de envío: 19/03/2017

Fecha de aceptación: 31/08/2017 


\section{INTRODUCCIÓN}

El incendio forestal es el fuego que se extiende sobre el terreno rústico, afectando a vegetación que no estaba destinada a arder (Vélez, 2003). Se considera un grave problema en España (WWF, 2015) porque supone un problema medioambiental y afecta a la integridad de las personas al localizarse a veces cerca de núcleos de población (Ponte y Bandín, 2008). Sotoca afirma que es la cuestión medioambiental que más preocupa a la población (APAS, 2003, 2004; IESA, 2008 citados en Sotoca, 2015) confirmándose en la última encuesta disponible de IESA (2013). Además, constituye un delito tipificado en el Código Penal Español en los artículos 352 a 355 del título XVII (Ley Orgánica 10/1995, de 13 de noviembre, actualizada en 2015) cuando se produce por causas humanas.

En España, se recoge información sobre este fenómeno desde el año 1968, con la Estadística General de Incendios Forestales (EGIF), administrada por la Dirección General de Desarrollo Rural y Política Forestal (Área de Defensa contra Incendios Forestales) del Ministerio de Agricultura y Pesca, Alimentación y Medio Ambiente (MAPAMA) (Sotoca, 2015). Los últimos avances informativos del MAPAMA muestran que en el año 2015 se produjeron 11928 incendios forestales y, según la Fiscalía General del Estado (2016), se identificaron sólo a 429 posibles autores, siendo la tasa de "esclarecimiento" excesivamente baja. En términos absolutos sería menor del $4 \%$, aunque la cifra aumentaría si se descontasen los producidos por fuerzas de la naturaleza, las reproducciones de otros incendios y aquellos en los que la intencionalidad sólo se pudo suponer. En cualquier caso, el incendio forestal sería uno de los fenómenos delictivos con menor tasa de esclarecimiento (Sotoca, González, Fernández, Kessel, Montesinos y Ruíz 2013). Para mejorar este porcentaje, durante el verano del año 2006 se inició una investigación sobre la caracterización psicosociológica del incendiario forestal, oficiada por la Fiscalía del Tribunal Superior de Justicia de Galicia primero y extendida a todo el territorio nacional después por la Fiscalía Coordinadora de Medio Ambiente y Urbanismo de la Fiscalía General del Estado (González, 2011; Fiscalía coordinadora de Medio Ambiente y Urbanismo, 2014). La Sección de Análisis del Comportamiento Delictivo (SACD) de la Unidad Técnica de Policía Judicial de la Guardia Civil asumió la dirección de esta iniciativa utilizando la técnica del perfilamiento criminal, obteniendo una tipología del incendiario forestal en tres grandes perfiles de incendio e incendiario forestal: "imprudente", "sin sentido" y por "obtención de beneficio" (Sotoca y cols., 2013; Sotoca, 2015). Ésta técnica ayuda a la investigación policial indicando qué tipo de persona habrá cometido con mayor probabilidad los hechos, según el modo en que los haya ejecutado (Douglas, Ressler, Burguess y Hartman, 1986; Hicks y Sales, 2006). Para inferir las características físicas, criminológicas y psicológicas de los individuos, la literatura diferencia dos grandes aproximaciones: la deductiva, que sólo tiene en cuenta la información disponible en el caso concreto que se esté estudiando (realizando predicciones sobre el autor teniendo en cuenta la evidencia conductual presente en fuentes de información como la escena del crimen, autopsia, victimología, variables espaciotemporales, etc.) y la inductiva, que se basa en la elaboración de tipologías tras el estudio de numerosos casos similares resueltos, de tal forma que posteriormente se puedan inferir características del autor de un delito sin esclarecer por comparación con otros que lo hayan ejecutado de manera similar (Canter, 2000; Muller, 2000; Ainsworth, 2001; Canter y Alison, 2003). Como referente y trabajo pionero en crear una tipología criminal del incendiario forestal, destaca la investigación del Instituto Superior de Policía Judiciária e Ciências Criminais y de la Universidad do Minho (Soeiro y Guerra, 2015) que ha inspirado las investigaciones realizadas en España. En la bibliografía no se encuentran más estudios centrados específicamente en el incendiario forestal, aunque sí se han realizado trabajos sobre perfiles de incendiarios en general (pero incluyendo en la misma muestra a personas que queman el monte, cubos de basura, edicicios, etc.)

Destacan en Australia, Willis (2004) con una extensa revisión de la literatura científica sobre los incendiarios y Kokcsis, que sigue la línea del perfilamiento inductivo en casos resueltos (Kocsis, Irwin y Hayes, 1998; Kocsis y Cooksey, 2002; Kocsis, 2004). En América, en Estados Unidos, Quinsey, Harris, Rice y Cormier (2006) analizaron el riesgo de reincidencia, y Douglas, Burguess, Burguess y Ressler (2006) aportaron una clasificación sobre las motivaciones. Dresdner (2014), desde un punto de vista clínico, estudió la presencia de trastornos en una muestra de 197 sujetos, imputados por distintos tipos de incendio en general, peritados en el Servicio Médico Legal Metropolitano de Chile durante el periodo de 1999 a 2012. 
González, J. L., Muñoz, V., Cacerrada, M. L. \& Sotoca, A. (2017). Perfil psicosocial del incendiario forestal español privado de libertad. Behavior \& Law Journal, 3(1), 26-34.

En Europa destacan Fritzon (2001) estudiando la distancia del domicilio del autor al incendio y las motivaciones; Gannon y Pina (2010) con un análisis de investigaciones recopiladas, llegando a la conclusión de tan sólo un pequeño porcentaje de incendios pueden ser atribuídos a pirómanos; Labree, Nijman, van Marle y Rassin (2010) que comparan las características de personalidad, consumo de sustancias, inteligencia y motivación de una muestra de incendiarios con otro tipo de delincuentes y trabajos más recientes como el de Thomson, Tiihonen, Miettunen, Sailas, Virkkunen y Lindberg (2015) con el objetivo de evaluar la existencia o no de rasgos de psicopatía y el de Ó Ciardha, Alleyne, Tyler, Barnoux, Mozova y Gannon (2015) que analizan la personalidad y psicopatología de una muestra de incendiarios en comparación con una muestra control. En España en concreto, destaca el trabajo de Heredia (2009) que revisa sentencias de tribunales españoles analizando las características de los implicados en delitos de incendio.

Dada la carencia de estudios científicos sobre los incendiarios forestales se hace necesario ahondar en esta materia siguiendo la línea de investigación abierta por la SACD en el año 2006, con el objetivo de ayudar en la investigación policial a identificar a un mayor número de autores analizando las características psicosociales de los responsables de incendio forestal que hayan sido privados de libertad, tratando de analizar las circunstancias que han podido influir en su detención. En el sistema penitenciario español se encuentran 68 Establecimientos Penitenciarios para el cumplimiento de penas, medidas de seguridad y prisión preventiva, 3 Centros Psiquiátricos Penitenciarios para la custodia y tratamiento de internos con trastornos graves de conducta que han sido internados por los Tribunales de Justicia y 13 Centros de Inserción Social para el cumplimiento de penas privativas en libertad en régimen abierto. En el momento de elaborar este texto hay 60220 reclusos (92.43\% hombres y $7.57 \%$ mujeres), de los cuales, 17147 son extranjeros. La mayor parte internos por delitos contra el Patrimonio y el Orden Socioeconómico seguido por delitos contra la Salud Pública (SGIPSecretaría General de Instituciones Penitenciarias, 2016).

En la investigación española se encuentran estudios sobre la personalidad de las personas privadas de libertad por otro tipo de delitos, por lo que se podrían comparar con las características de los incendiarios forestales. Romero, Luengo y Sobral (2001) estudiaron en Galicia las variables de extraversión, neuroticismo, psicoticismo, impulsividad y búsqueda de sensaciones en 435 adolescentes varones escolarizados, 529 adolescentes mujeres escolarizadas y 95 adolescentes varones delincuentes institucionalizados en centros de rehabilitación juvenil o prisiones, encontrándose diferencias significativas entre los chicos escolarizados y los chicos institucionalizados, presentado estos últimos mayores puntuaciones en neuroticismo, psicoticismo, impulsividad y en dos componentes de búsqueda de sensaciones (búsqueda de experiencia y susceptibilidad de aburrimiento), y menores en extraversión. Rodríguez, López y Pueyo (2002) examinaron la impulsividad, temeridad, empatía, hostilidad y estabilidad emocional en 99 reclusos varones por múltiples delitos o delitos graves, en un centro penitenciario de Cataluña en el periodo 19971998 que mostraban puntuaciones altas en impulsividad, temeridad y agresividad-hostilidad (comparadas con los valores de referencia en poblaciones no delincuentes), más bajas en empatía y deseabilidad social, y similares en neuroticismo. Ortiz-Tallo, Fierro, Blanca, Cardenal y Sánchez (2006) no encuentran diferencias significativas en una muestra de 54 reclusos de la prisión de Málaga (51 varones y 3 mujeres) por delitos violentos, comparados con 57 sujetos normales, utilizando el Big Five Questionnaire (BFQ), excepto en el factor energía (similar a extraversión), al presentar un nivel menor. Sobral, Luengo, Gómez-Fraguela, Romero y Villar (2007) en una muestra de 216 sujetos (108 hombres y 108 mujeres) en centros penitenciarios gallegos y en dos catalanes, agrupados en una categoría de delitos violentos (124 asesinatos, homicidios, detenciones ilegales, agresiones sexuales, robo con violencia, lesiones y coacciones) y otra de no violentos (92 delitos contra la salud pública, hurto, falsedad y estafa) examinaron el factor de Retraimiento Social (relacionado con las escalas de impulsividad, agresión, hostilidad, sinceridad, autoestima) y el factor de Beligerancia (relacionado con las escalas de timidez, introversión, ansiedad y depresión), obteniendo como resultado que las mujeres encarceladas tenían niveles de autoestima significativamente más bajos, mayores puntuaciones en evitación, y menores niveles de extraversión que los hombres encarcelados. Respecto al tipo de delito, los delincuentes no violentos presentaban mayor autocontrol y autoestima y menores puntuaciones en evitación, recelo paranoide, resentimiento, desviación, y extraversión. Herrero y Colom (2008) no hallaron diferencias en extraversión entre 183 varones internos en dos cárceles de la Comunidad de Madrid y 397 varones de 
la población en general, pero sí en psicoticismo y neuroticismo, obteniendo mayores puntuaciones los presos. Similares resultados tuvieron Ferrer, Carbonell, Sarrado, Cebrià, Virgili y Castellana (2010) comparando 42 infractores institucionalizados adolescentes con 86 estudiantes.

Siguiendo esta línea de estudios,, este trabajo trata de dar un paso más en la investigación sobre los incendiarios forestales, centrándose en el estudio de algunas características psicosociales de los autores privados de libertad, viendo si es posible identificar variables psicológicas (de personalidad e inteligencia) que puedan responder a por qué han llegado a esa situación frente a muchos otros que no son ni siquiera identificados, o que siéndolo no son condenados o privados de libertad por ello. Se analizará cuánto se distinguen los incendiarios que han llegado a estar en prisión, de los que solamente fueron identificados policialmente y analizados en el estudio de Sotoca (2015). Se pretende ayudar así a las Fuerzas y Cuerpos de Seguridad del Estado a mejorar la investigación criminal, visibilizando los casos que han pasado por prisión, para que se recopilen mayores indicios en el resto de perfiles. A la vez, se tratará de comparar las características de personalidad de los incendiarios forestales privados de libertad con el resto de población carcelaria, analizando similitudes y diferencias, por si se pudiese aislar un patrón distintivo en los incendiarios.

\section{MÉTODo}

\section{A. Muestra}

Instituciones Penitenciarias identificó a 50 personas privadas de libertad en el periodo 2008-2013 (22 preventivas y 28 penadas cumpliendo sentencia firme), consideradas responsables de un delito de incendio forestal. Del total, 43 sujetos estaban en 26 establecimientos penitenciarios de todo el territorio nacional y 7 sujetos en 3 centros psiquiátricos (Alicante, Ourense y Santiago de Compostela). Todos eran de nacionalidad española y varones, excepto dos mujeres y con edades comprendidas entre 19 y 71 años (media: 43.10; desviación típica: 13.91). A estas personas se les habían adjudicado 114 incendios producidos en el periodo 1993-2013.

\section{B. Instrumentos}

Para recoger los datos sobre los incendios y las características psicosociales de sus autores, se utilizó un cuestionario desarrollado ad hoc por la Sección de Análisis del Comportamiento delictivo (SACD) de la Guardia Civil, donde se examinaban las variables que se reflejan en las tablas 1 y 2. Para la evaluación de la personalidad, se utilizó el EPQ-R (Eysenck y Eysenck, 2001), muy utilizado en el contexto criminal y con buenas propiedades psicométricas, ofreciendo una medida de la personalidad muy ligada a factores temperamentales. Para contar con una medida de inteligencia, se eligió el test de Matrices Progresivas de Raven (Raven, Raven y Court, 1993), que debido a su contrastado desarrollo metodológico y sus condiciones de aplicabilidad (brevedad, existencia de diferentes escalas que cubren un amplio espectro de sujetos en cuanto a dotación intelectual y tarea libre de condicionamientos culturales) se ajustaba muy bien a las restricciones de la entrevista en el contexto penitenciario.

\section{Procedimiento}

A medida que se identificaban personas privadas de libertad por incendio forestal, la Fiscalía de Medio Ambiente solicitaba autorización al establecimiento para realizar una entrevista con el sujeto, voluntaria y previo consentimiento informado. En todos los casos el sujeto accedió y al menos dos psicólogos criminalistas de la SACD se desplazaban a la prisión y recababan información del expediente, las impresiones de los profesionales que les atendían, cumplimentaban el cuestionario psicosocial a partir de la entrevista personal y aplicaban las pruebas de personalidad e inteligencia.

Recogida la información, las preguntas del cuestionario psicosocial y los resultados de las pruebas psicológicas se convirtieron en variables objeto de estudio, elaborándose una base de datos anónima, la cual fue cedida para el presente estudio con el fin de analizarla a través del programa SPSS versión 19, aplicando estadísticos descriptivos. Se contó con 24 variables sobre los incendios (tabla 1) y 42 variables sobre los autores (tabla 2). 
González, J. L., Muñoz, V., Cacerrada, M. L. \& Sotoca, A. (2017). Perfil psicosocial del incendiario forestal español privado de libertad. Behavior \& Law Journal, 3(1), 26-34.

Tabla 1. Variables del hecho

\begin{tabular}{ll}
\hline \hline Comunidad Autónoma & Número de focos \\
\hline Quincena & Punto de inicio \\
\hline Mes & Tipo de superficie \\
\hline Estación & Uso principal \\
\hline Año & Medio de ignición \\
\hline Día de la semana & Ayuda en extinción \\
\hline Hora de inicio & Víctimas \\
\hline Nivel de riesgo & Atribución motivación \\
\hline Denunciante & Motivo: beneficio \\
\hline Autor visto & Motivo: daños a terceros \\
\hline Delito asociado & Motivo: conductas anómalas \\
\hline Delito específico & Motivo: imprudencia \\
\hline \hline
\end{tabular}

Tabla 2. Variables del autor

\begin{tabular}{ll}
\hline \hline Edad & $\begin{array}{l}\text { Localización de incendio } \\
\text { respecto del domicilio }\end{array}$ \\
\hline Sexo & $\begin{array}{l}\text { Distancia entre incendio y } \\
\text { domicilio }\end{array}$ \\
\hline Nacionalidad & $\begin{array}{l}\text { Localización incendio respecto } \\
\text { del trabajo }\end{array}$ \\
\hline Estado Civil & Conoce al propietario \\
\hline Situación laboral & Relación con el propietario \\
\hline Trabajo principal & Actitud ante la detención \\
\hline Sector laboral & Atribución de motivación por \\
\hline Tipo de trabajo & autor \\
\hline Asistencia al trabajo & Asume la responsabilidad \\
\hline Adaptación al trabajo & Medio de transporte \\
\hline Franja de ingresos & Cómplices \\
\hline Nivel educativo & Vigilancia policial \\
\hline Infancia & Detenciones anteriores \\
\hline Crianza & Incendios en serie \\
\hline Estilo de vida & Situación penal \\
\hline Residencia & Centro donde cumple condena \\
\hline Relaciones sociales & Tiempo condenado \\
\hline Tiempo libre & Sanciones \\
\hline Tratamiento psicológico & Inteligencia \\
\hline Diagnóstico principal & Extraversión (EPQ- R) \\
\hline Otros problemas de salud & Psicoticismo (EPQ-R) \\
\hline Abuso de sustancias & Neuroticismo (EPQ-R) \\
\hline Tipo de sustancia & Sinceridad (EPQ-R) \\
\hline \hline
\end{tabular}

\section{RESULTADOS}

\section{A. Variables del becho}

Se analizan las variables de los 114 incendios forestales pertenecientes a los 50 autores. Los años 2007, 2009 y 2013 cuentan con mayor número de incendios provocados por autores privados de libertad (24.24\%, 19.3\% y 16.7\% respectivamente). Como Comunidad Autónoma destaca Galicia (46.5\%) seguida de Madrid (17.5\%) y Castilla y
León (15.8\%). Julio y agosto fueron los meses con mayor número de incendios $(55.2 \%)$, no produciéndose ninguno en noviembre y diciembre y siendo más frecuente como periodo del día las tardes (de 15 a 20 horas; 40.4\%). Fueron más comunes las denuncias por vecinos $(37.7 \%)$ y se vio al autor en el $50.9 \%$ de los incendios. No fue frecuente la existencia de delitos asociados al incendio, sólo en 3 de los incendios registrados, siendo éstos delito de amenazas, delito de daños y robo y delito de robo de cobre. Gran parte de los incendios se iniciaron con un mechero (69.3\%) mediante un único foco (66.7\%) siendo habitual, en los casos en los que se conoce, que comenzaran en pista forestal $(24.6 \%)$ o en camino $(12.3 \%)$, que el tipo de superficie más común fuera la masa forestal $(21.9 \%)$ o matorral $(17.5 \%)$ y el uso principal de la zona afectada el de aprovechamiento forestal (33.3\%). En la mayor parte de los incendios no hubo víctimas, sólo en 6 de ellos, aunque el $42.1 \%$ del total de los incendios ha sido calificado de riesgo alto. En cuanto a la motivación atribuida a cada incendio se puede observar en la figura 1, en la que destaca que el mayor porcentaje de incendios se clasifican como debidos a "conductas anómalas", que suelen ser sin sentido y mayoritariamente a cargo de sujetos con algún tipo de problema psicológico o psiquiátrico (ver figura 2).

Figura 1. Porcentajes de las motivaciones atribuidas por los investigadores a cada incendio forestal.

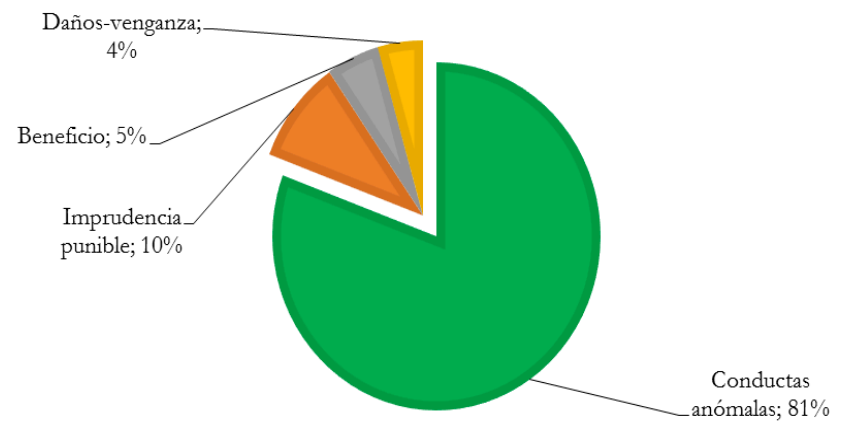

\section{B. Variables del autor}

Se analizan los 50 autores responsables de los incendios. El 56\% se encontraban casados o con pareja, viviendo acompañados más de la mitad $(60 \%)$ ya fuera con los padres, pareja u otras personas. El 80\% tenía algún tipo de empleo, ya fuera esporádico o no, o era pensionista o jubilado y fundamentalmente trabajaban en el sector agrícola o en la construcción. Sólo 4 de los sujetos tenían 
González, J. L., Muñoz, V., Cacerrada, M. L. \& Sotoca, A. (2017). Perfil psicosocial del incendiario forestal español privado de libertad. Behavior \& Law Journal, 3(1), 26-34.

salarios por encima de 1200 euros al mes. Un 16\% de los autores eran analfabetos y sólo un 10\% llegó a estudiar bachillerato o en la universidad. La mayoría de los sujetos (78\%) manifestaron tener una infancia normal. El 70\% de los sujetos vivían en entornos rurales y la mayoría de los domicilios de los autores se situaban cerca del lugar del incendio (74\%), desplazándose al lugar del incendio a pie (64\%). El 58\% de los incendiarios no conocían al propietario de los terrenos y 2 eran ellos mismos los propietarios. Sólo un $22 \%$ del total asumían la responsabilidad del fuego y del incendio. La gran mayoría actuaron solos $(94 \%)$ y tenían detenciones anteriores por otros delitos el 56\%. El 40\% de los autores llevaron a cabo incendios en serie. El 52\% de los sujetos alegaban preferir pasar el tiempo en soledad y el $26 \%$ manifestaban no tener amigos.

Analizando el historial clínico y psicológico de los sujetos se encuentra que el 58\% había recibido tratamiento psicológico o psiquiátrico previo, y constaban como diagnósticos psicológicos principales los que se presentan en la figura 2. Además, el $74 \%$ de los sujetos presentaban abuso de sustancias, destacando el consumo de alcohol $(\mathrm{N}=37 ; 59.5 \%)$

Figura 2. Diagnóstico psicológico principal de los sujetos de la motivación "conductas anómalas".

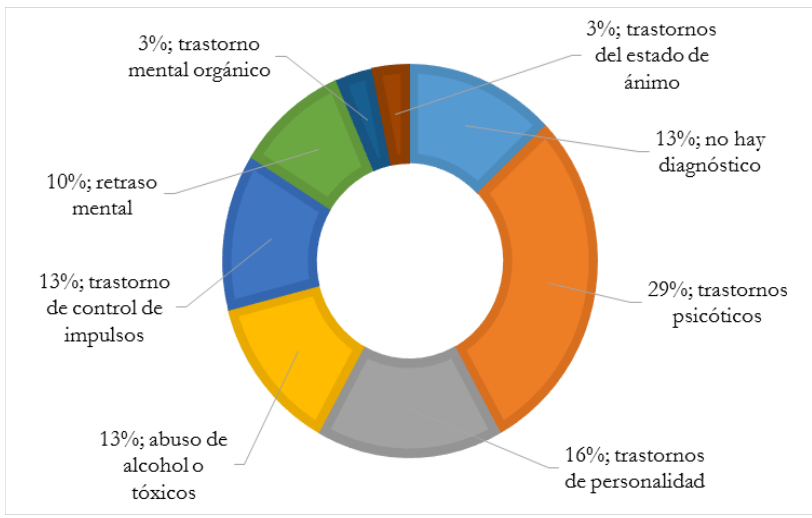

Respecto a las variables de inteligencia, se descartó a dos sujetos por no cumplimentar de forma correcta el cuestionario y, en las variables de personalidad, se evaluó sólo a 24 sujetos (23 en psicoticismo por la misma razón), que fueron los que se consideraron válidos en la escala de sinceridad (para la evaluación fueron descartados siete sujetos igualmente por no cumplimentar el cuestionario de forma correcta). Se puede ver el porcentaje de sujetos por puntuaciones en la tabla 4.
Tabla 3. Frecuencias y porcentajes de las variables de personalidad e inteligencia y escala de sinceridad.

\begin{tabular}{lcc}
\hline \hline Variables & $\mathrm{n}$ & $\%$ \\
\hline \hline Inteligencia (N=48) & 34 & 70.8 \\
Baja (CI menos 80)) & 14 & 29.2 \\
Media (CI 80 a 110) & 0 & 0 \\
Alta (CI más de 110) & & \\
\hline Nivel de extraversión (N=24) & 9 & 37.5 \\
Baja (menos de 40) & 11 & 45.8 \\
Media (entre 40 y 60) & 4 & 16.7 \\
Alta (más de 60) & & \\
\hline Emotividad-Neuroticismo (N=24) & 6 & 25 \\
Baja (menos de 40) & 11 & 45.8 \\
Media (entre 40 y 60) & 7 & 29.2 \\
Alta (más de 60) & & \\
\hline Dureza-Piscotisimo (N=23) & 2 & 8.7 \\
Baja (menos de 40) & 15 & 65.2 \\
Media (entre 40 y 60) & 6 & 26.1 \\
Alta (más de 60) & & \\
\hline Sinceridad (N=50) & 24 & 48 \\
Vale (menos de 60) & 19 & 38 \\
No vale (más de 60) & 7 & 14 \\
No se sabe & & \\
\hline \hline
\end{tabular}

\section{DISCUSIÓN}

$\mathrm{El}$ incendio forestal es un grave problema que se repite año tras año en España quemando gran cantidad de hectáreas, constituyendo un peligro para el medio ambiente y las personas. Es además un fenómeno delictivo necesitado de nuevas técnicas que aumenten su tasa de esclarecimiento, por lo que la SACD de Guardia Civil lleva años realizando estudios sobre perfilado para mejorar esas cifras. Pero hasta el momento todos los trabajos en España utilizaban muestras de detenidos e investigados policialmente, no habiendo profundizado en los que llegan efectivamente a estar privados de libertad, por lo que el presente estudio constituye una investigación pionera.

En cuanto a los resultados, habría que destacar que los incendiarios forestales privados de libertad inician los fuegos con mayor frecuencia como consecuencia de trastornos psicopatológicos, es decir se ajustarían a la tipología de incendiario de "conductas anómalas". En cambio, los incendiarios que con mayor porcentaje identifican las Fuerzas y Cuerpos de Seguridad son los que cometen los hechos por una imprudencia. De este hallazgo se podría concluir que la mayoría de personas que son detenidas o investigadas por incendio forestal no terminan en prisión, puesto que sólo un $10 \%$ de los incendiarios que se encuentran privados de libertad han cometido los hechos por imprudencia grave. Otra conclusión que podría 
extraerse de los resultados es que probablemente las características psicológicas de las personas que se encuentran en prisión facilitaron su identificación policial, al presentar la mayoría un trastorno psicológico y/o un nivel de inteligencia bajo, es decir carentes de habilidades para evitar con éxito la acción policial.

Los acusados por incendio forestal que llevaron a cabo los hechos por venganza o por obtención de algún beneficio son muy escasos, dato que corrobora los resultados previos (Sotoca, 2015). Para conocer si realmente estas motivaciones son infrecuentes o es que no se identifica a los responsables y, por tanto, no ingresan en prisión, sería necesario analizar los incendios forestales sin esclarecer, pero cuya motivación se pudo suponer, para observar si estas motivaciones aumentan o no, lo que se abordará en futuros estudios.

Profundizando en los diagnósticos psicopatológicos de los incendiarios que se encuentran en prisión, los datos confirman lo hallado en otros estudios (Gannon y Pina, 2010), en cuanto a que la piromanía es una condición excepcional y que es más probable que este tipo de autores tengan otro tipo de patología psicológica, pese a lo frecuente en los medios de comunicación de equiparar incendiario a pirómano. Como el mayor porcentaje de la muestra de este estudio se ajustaría a la tipología "conductas anómalas", era esperable que la mayoría de autores consumieran algún tipo de sustancia, tal y como se encontró en Sotoca (2015), siendo el alcohol la más predominante, algo coincidente también en el estudio portugués de Soeiro y Guerra (2015). No obstante, esto no sería prototípico sólo de los incendiarios, puesto que un alto porcentaje de población carcelaria abusa de algún tipo de sustancia (Rodríguez et al., 2002; Ortiz-Tallo et al., 2006; Ferrer et al., 2010).

También era esperable que el perfil genérico de incendiario forestal propuesto en Sotoca (2015) fuese distinto al encontrado en este trabajo, al estar aquel sobrerrepresentado de incendios imprudentes y éste de "conductas anómalas". No extraña, por tanto, que variables de tipo antisocial e indicativas de desadaptación personal y social estén más presentes en esta muestra. Así, la mayoría de incendiarios que son identificados por las Fuerzas y Cuerpos de Seguridad están casados, prefieren estar con gente en su tiempo libre, no tienen antecedentes policiales ni han estado en tratamiento psicológico. Pero los que pasan por establecimientos penitenciarios no suelen vivir en pareja en el momento de los hechos, la mitad prefiere estar sólo en su tiempo libre, tienen antecedentes policiales y la mayoría han estado en tratamiento psicológico, fundamentalmente por trastornos psicóticos.

Con respecto a las características de personalidad de los incendiarios forestales privados de libertad en comparación con el resto de población carcelaria, la primera conclusión que se puede obtener es que los instrumentos de autoinforme presentan limitaciones para su uso en población forense, por los sesgos de respuesta, habiendo tenido que eliminar más del 50\% de la muestra por riesgo de falta de sinceridad debido a fenómenos de deseabilidad social. Este tipo de hallazgos refuerza la idea de que deben desarrollarse protocolos de perfilado indirecto de personalidad, tal y como han propuesto González, Sotoca y Garrido (2015) o González y López (2016). No obstante, de los sujetos que pudo obtenerse información fiable llama la atención que la mayoría ha obtenido puntuaciones medias en los rasgos de extraversión, neuroticismo y psicoticismo, por lo que el incendiario forestal privado de libertad no contaría con ningún rasgo desviado de la población general, ni por exceso ni por defecto. En cualquier caso, esto no difiere demasiado de las investigaciones al respecto en población penitenciaria: La variable extroversión sólo se ha asociado a delincuencia violenta (Sobral et al., 2007), siendo no significativa en el resto de población penitenciaria (Romero et al., 2001; Herrero et al., 2008; Ferrer et. al., 2010) o incluso con cierta tendencia a la introversión (Ortiz-Tallo et. al., 2006). Resultados similares se han obtenido con el neuroticismo y psicoticismo, encontrando estudios que sí han correlacionado delincuencia con neuroticismo (Herrero et al., 2008; Ferrer et. al., 2010) o psicoticismo (Romero et. al., 2001; Rodríguez et. al., 2002; Herrero et al., 2008; Ferrer et. al., 2010) mientras que otros han obtenido puntuaciones similares a la población general (Romero et. al., 2001; Rodríguez et. al., 2002; Ortiz-Tallo et. al., 2006;). Puede concluirse, por tanto, que no hay acuerdo en la literatura científica sobre si algún rasgo de personalidad del modelo PEN (Eysenck y Eysenck, 2001) está más asociado a la delincuencia española o no, pero en cualquier caso parece que el incendiario forestal no es muy distinto del resto de población penitenciaria ni de la población general.

Por último, es preciso señalar que este estudio no está exento de limitaciones, especialmente en lo que tiene que ver con el tamaño de la muestra. Aunque se han incluido a 
González, J. L., Muñoz, V., Cacerrada, M. L. \& Sotoca, A. (2017). Perfil psicosocial del incendiario forestal español privado de libertad. Behavior \& Law Journal, 3(1), 26-34.

todos los incendiarios forestales que se ha podido constatar que se encontraban en prisión durante los años 2008-2013, probablemente el número real sea mayor. Además fue necesario eliminar a más del 50\% de esta muestra para la medida de personalidad, por no haber cumplimentado el test de manera correcta o por riesgo de falta de sinceridad. Todo esto imposibilita que los resultados de este estudio puedan generalizarse. No

\section{REFERENCIAS}

Ainsworth, P.B. (2001). Offender profiling and crime analysis (1st ed.). Cullompton, UK: Willan.

APAS - Asociación para la Promoción de Actividades Socioculturales e Instituto de Estudios del Medio, S.L. (2003). Estudio sociológico sobre la percepción de la población española hacia los incendios forestales. Recuperado de: http://www.idem21.com/descargas/pdfs/IncendiosForestales.pdf

APAS- Asociación para la Promoción de Actividades Socioculturales- e Instituto de Estudios del Medio, S.L. (2004). Estado del conocimiento sobre las causas de los incendios forestales en España. Recuperado de

http://www.idem21.com/descargas/pdfs/CAUSAS_IF.pdf

Canter, D. (2000). Offender profiling and criminal differentiation. Legal and Criminological Psychology, 5, 23-46.

Canter, D. y Alison, L.J. (2003). Converting evidence into data: The use of law enforcement archives as unobtrusive measurement. The Qualitative Report, 8 (2), 151-176.

Douglas, J.E., Burguess, A.W., Burguess A.G., y Ressler, R.K. (2006). Crime Classification Manual: A Standard System for Investigating and Cassifying Violent Crime (2nd ed.) (pp. 261-291) San Francisco, Calif:: Jossey-Bass.

Douglas, J. E., Ressler, R.K., Burgess, A.W. y Hartman, C.R. (1986). Criminal profiling from crime scene analysis. Behavioral Sciences \&o the Law, 4, 401-421.

Dresdner, C. (2014). Perspectiva psiquiátrico forense en incendiarios. (Tesis doctoral), Facultad de Ciencias Médicas, Universidad Nacional de La Plata, Santiago, Chile- La Plata, Argentina. Recuperado de http://sedici.unlp.edu.ar/handle/10915/40284

Eysenck, H.J. y Eysenck, S.B.G. (2001). EPQ - R. Cuestionario revisado de Personalidad de Eysenck. Madrid: TEA Ediciones.

Ley Orgánica 10/1995, de 23 de Noviembre, del Código Penal. Boletín Oficial del Estado, núm. 281, de 24 de Noviembre de 1995, pp. 130. https://boe.es/buscar/act.php?id=BOE-A-1995$25444 \& \mathrm{p}=20150428 \& \operatorname{tn}=2$

España. Ministerio del Interior - Secretaría General de Instituciones Penitenciarias (2016). Estadística penitenciaria mensual de septiembre. Recuperado de: http://www.institucionpenitenciaria.es/web/portal/administracion Penitenciaria/estadisticas.html

Ferrer, M., Carbonell, X., Sarrado, J. J., Cebrià, J., Virgili, C., y Castellana, M. (2010). Distinguishing male juvenile offenders through personality traits, coping strategies, feelings of guilt and obstante, sí que constituiría un primer acercamiento al perfil de incendiario forestal privado de libertad y en estudios posteriores se comprobará si se mantienen o no las tendencias encontradas. Así pues, el que no haya emergido un perfil prototípico de personalidad, no quiere decir que no pueda aparecer en el futuro y la creciente necesidad de su realización.

level of anger. The Spanish journal of psychology, 13(02), 751-764.

Fiscalía Coordinadora de Medio Ambiente y Urbanismo (2014). Elaboración del perfil psicosocial del incendiario forestal. Informe 2014. En Fiscalía Coordinadora de Medio Ambiente y Urbanismo (Ed.), Memoria 2014. Madrid: Editor

Fiscalía General del Estado (2016). Memoria 2016. Madrid: Editor. Recuperado de

https://www.fiscal.es/memorias/memoria2016/Inicio.html

Fritzon, K. (2001). An examination of the relationship between distance traveled and motivational aspects of firesetting behaviour. Journal of Environmental Psychology, 21, 45 - 60.

Gannon, T. y Pina, A. (2010). Firesetting: Psychopathology, theory and treatment. Aggression and Violent Behavior, 15, 224-238.

González, J. L. (2011). El perfil psicosocial del incendiario forestal. Boletín de la Asociación de Técnicos de Instituciones Penitenciarias, 25, 4-10.

González, J. L., Sotoca, A. y Garrido, M. J. (2015). El perfilamiento en la investigación criminal. En Giménez y González (Coords.), Investigación Criminal: principios, técnicas y aplicaciones. (1 $1^{\mathrm{a}} \mathrm{ed}$.) (pp. 211224). Madrid: LID Editorial Empresarial, S.L.

González, J. L. y López, R. (2016). Personalidad y comportamiento. Perfilación Indirecta de Personalidad. En López, R., Gordillo, F. y Grau, M. (Coords.), Manual de Análisis de Comportamiento no Verbal: Más allá de la comunicación y el lenguaje. (131-153). Madrid: Pirámide.

Heredia, F. (2009). Pirómanos e incendiarios. Estudio psiquiátrico forense. Revista La Ley Penal, 66, 106-119.

Herrero, O. y Colom, R. (2008). Distinguishing Impulsive, Unsocialized Sensation Seeking. A comparison between Criminal Offenders and the General Population. Journal of Individual Differences, 29 (4), 199204.

Hicks, S. J., y Sales, B. D. (2006). Criminal profiling: Developing an effective science and practice. Washington, DC: American Psychological Association.

IESA- Instituto de Estudios Sociales de Andalucía, y CSIC - Consejo Superior de Investigaciones Científicas (2008). Ecobarómetro de Andalucia 2008 - Documento de sintesis. Recuperado de :http://www.juntadeandalucia.es/medioambiente/site/portalweb/ menuitem.7e1 cf46ddf59bb227a9ebe205510e1ca/?vgnextoid $=92 \mathrm{c} 12$ 42c76565410VgnVCM2000000624e50aRCRD\&vgnextchannel=f83 bc17a45b05310VgnVCM1000001325e50aRCRD\&lr=lang_es 


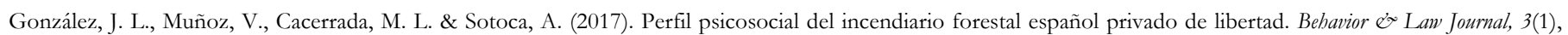
26-34.

IESA- Instituto de Estudios Sociales de Andalucía, y CSIC - Consejo Superior de Investigaciones Científicas (2013). Ecobarómetro de Andalucía 2013 - Documento de sintesis. Recuperado de

http://www.juntadeandalucia.es/medioambiente/portal_web/servicios _generales/doc_tecnicos/2013/ecobarometro_2013_sintesis/sintes is $2013 \mathrm{html} /$ index.html\#6

Kocsis, R.N. (2004). Psychological Profiling of Serial Arson Offenses: An Assessment of Skills and Accuracy. Criminal Justice and Behavior, 31, (3) $341-361$.

Kocsis, R. N., Cooksey, R. W. (2002). Criminal psychological profiling of serial arson crimes. International Journal of Offender Therapy and Comparative Criminology, 46(6), 631-656.

Kocsis, R.N., Irwin, H.J., y Hayes, A.F. (1998). Organised and Disorganised Criminal Behaviour Syndromes in Arsonists: A Validation Study of a Psychological Profiling Concept. Psychiatry, Psychology and Law, 5, 117 - 131.

Labree, W., Nijman, H., van Marle H. y Rassin, E. (2010). Backgrounds and characteristics of arsonists. International Journal of Law and Psychiatry 33, 149-153.

MAPAMA - Ministerio de Agricultura y Pesca, Alimentación y Medio Ambiente (2015). Los Incendios Forestales en España: Avance Informativo. 1 de enero al 31 de Diciembre del 2015. Recuperado de

http://www.magrama.gob.es/es/desarrollo-

rural/estadisticas/avanceinformativo1deeneroa31dediciembrede201 5_tcm7-411417.pdf

Muller, D. A. (2000). Criminal profiling. Real science or just wishful thinking? Homicide Studies, 4, 234-264.

Ó Ciardha, C., Alleyne, E.K.A., Tyler, N., Barnoux, M.F.L., Mozova, K., y Gannon, T.A. (2015). Examining the Psychopathology of Incarcerated Male Firesetters using the MCMI-III. Psychology, crime and Law, 21 (6), 606-616.

Ortiz-Tallo, M., Fierro, A., Blanca, M.J., Cardenal, V., y Sánchez, L.M. (2006). Factores de personalidad y delitos violentos. Psicothema, 18, 459-464.

Ponte, J.M. y Bandín, C. (2008). Los incendios forestales en Galicia y su investigación. Estudios penales y criminológicos, 28, 317-341.
Quinsey, V. L., Harris, G. T., Rice, M.E. y Cormier, C. A. (2006). Fire Setters. En Violent offenders: Appraising and managing risk (2nd ed.) (115-129). The law and public policy. Washington, DC, US: American Psychological Association.

Raven, J., Raven, J.C. y Court, J. H. (1993). Test de Matrices Progresivas. Manual. Buenos Aires: Paidós.

Rodríguez, A., López, J. M. y Pueyo, A. A. (2002). Personalidad y comportamiento penitenciario. Psicothema, 14, 90-100.

Romero, E., Luengo, M.A. y Sobral, J. (2001). Personality and antisocial behaviour: study of temperamental dimensions. Personality and Individual Differences, 31, 329-348.

Sobral, J., Luengo, A., Gómez-Fraguela, J.A., Romero, E. y Villar, P. (2007). Personalidad, género y criminalidad violenta en reclusos. Psicothema, 19, 269-275.

Soeiro, C., y Guerra, R. (2015). Forest arsonists: criminal profiling and its implications for intervention and prevention. European police science and research bulletin 11, 34-40

Sotoca, A. (2015). Perfil criminológico del incendiario forestal: estudio empírico basado en la evidencia. (Tesis doctoral). Facultad de psicología. Universidad Complutense de Madrid, Madrid, España.

Sotoca, A., González, J. L., Fernández, S., Kessel, D., Montesinos, O., y Ruíz, M. Á. (2013). Perfil del incendiario forestal español: aplicación del perfilamiento criminal inductivo. Anuario de Psicología Jurídica, 23(1), 31-38.

Thomson, A., Tiihonen, J., Miettunen, J., Sailas, E., Virkkunen, M., y Lindberg, N. (2015). Psychopathic traits among a consecutive sample of Finnish pretrial fire-setting offenders. BMC Psychiatry, 15, [44]

Vélez, R. (2003). La defensa contra incendios forestales. Fundamentos $y$ experiencias. Madrid: McGraw-Hill.

Willis, M. (2004). Bushfire Arson: A Review of the Literature. Australian Institute of Criminology. Research and Public Policy Series, $\mathrm{N}^{\circ} 61$.

WWF - World Wildlife Fund (2015). Bosques listos para arder. Informe de $2015 . \quad$ Recuperado de: http://www.wwf.es/que_hacemos/bosques/problemas/incendios_ forestales/ 